\title{
FLEXIBILITY OF LHC OPTICS FOR FORWARD PROTON MEASUREMENTS*
}

\author{
KrZysztof CieŚlA
}

\author{
Faculty of Physics, Mathematics and Computer Science \\ Cracow University of Technology \\ Podchorążych 1, 30-084 Kraków, Poland \\ RAFAє StAsZEWSKI
}

The Henryk Niewodniczański Institute of Nuclear Physics

Polish Academy of Sciences

Radzikowskiego 152, 31-342 Kraków, Poland

(Received April 29, 2016)

The geometric acceptance of the ATLAS Forward Proton detectors is studied. The elements of the LHC magnetic lattice that are most important for the acceptance are identified. The effects of possible changes of the LHC optics are studied.

DOI:10.5506/APhysPolB.47.1633

\section{Diffractive processes in hadron interactions}

One of the goals of the physics programme of the Large Hadron Collider [1] is the measurement of diffractive processes. These processes take place in proton-proton interactions as a result of the exchange of a colour singlet (Pomeron). Two signatures are typically used for experimental measurements of diffraction, namely the presence of a large rapidity gap or an intact forward proton. The intact protons are scattered at very small angles, which makes their detection by central detectors impossible. Measurements of these protons can only be performed at a large distance from the interaction point and very close to the centre of the beam. This can be achieved

\footnotetext{
* Presented at the Cracow Epiphany Conference on the Physics in LHC Run 2, Kraków,
} Poland, January 7-9, 2016. 
with help of the dedicated forward proton detectors. At the LHC, several systems of such detectors are installed, e.g. TOTEM [2], CT-PPS [3], ALFA [4] and AFP [5]. The results presented in this paper concern the AFP detectors; however, they are also partially applicable to TOTEM horizontal detectors.

\section{AFP detectors programme}

The goal of the AFP (ATLAS Forward Proton) detectors is the measurement of very forward protons, which are outside the central detector acceptance. To achieve this, the detectors are located at 205 and $217 \mathrm{~m}$ away from the interaction point, on each outgoing beam. Each of the four stations contains four planes of 3D Silicon pixel detectors [5]. In addition, the outer $(217 \mathrm{~m})$ stations are foreseen to be equipped with time-of-flight detectors. The AFP detectors will operate a few millimetres away from the centre of the beam. They will be placed in the vicinity of the beam using the Roman Pot technology.

The diffractive protons can be described with three kinematic variables; a typical choice is: the transverse momentum $p_{\mathrm{T}}$, the azimuthal angle $\varphi$ and the relative energy loss $\xi$, defined as: $\xi=\left(E_{\text {beam }}-E_{\text {proton }}\right) / E_{\text {beam }}$, where $E_{\text {beam }}$ is the beam energy and $E_{\text {proton }}$ is the energy of the scattered proton.

In the context of the detectors approaching the LHC beam in the horizontal direction and for the low- $\beta^{*}$ optics of the machine, the variable relevant for the acceptance is $\xi$. The kinematic region in which the diffractive protons are produced depends on the actual process. For soft single diffractive dissociation, the distribution has an approximate $1 / \xi$ shape, peaking strongly at low $\xi$ values. On the other hand, for diffractive processes in which a hard object is produced, the shape is much flatter. This is illustrated in Fig. 1, where the $\xi$ distributions are shown for soft single diffraction and several hard diffractive processes. The distribution for the $W$-boson production is steep, but not as much as for soft processes. The distributions for jets with $p_{\mathrm{T}}>20 \mathrm{GeV}$ is even flatter, while for jets with $p_{\mathrm{T}}>50 \mathrm{GeV}$, the distribution decreases with $\xi$ approaching zero.

The trajectory of a diffractively scattered proton through the LHC magnetic lattice ${ }^{1}$ depends on the accelerator optics and the particle momentum. The so-called proton transport can be simulated using a dedicated software, e.g. the MAD-X programme [6]. Figure 2 presents the projections of trajectories at the $(x, z)$ plane calculated for protons with different values of the relative energy loss and with the transverse momentum set to zero. The plot shows also positions of the elements of the LHC magnetic structure and the location of the AFP inner station.

\footnotetext{
${ }^{1}$ The composition of the accelerator magnets.
} 


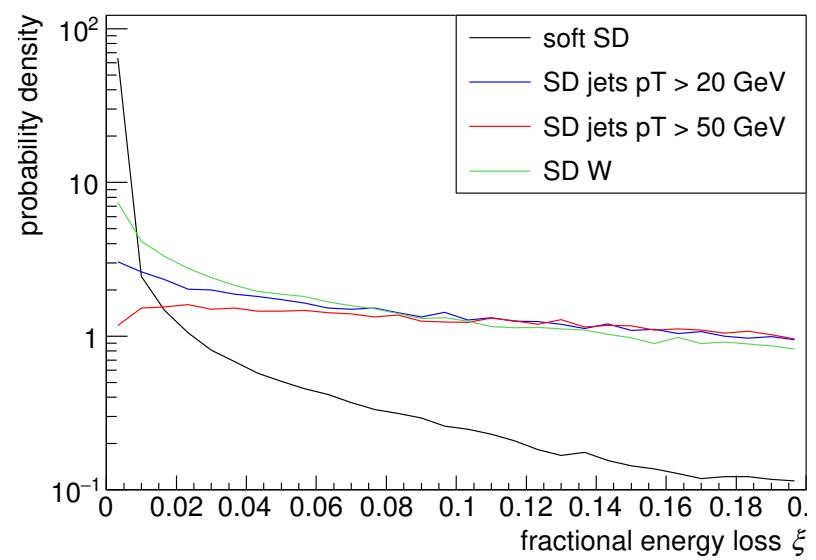

Fig. 1. Distributions of fractional energy loss for soft single diffractive dissociation, single diffractive $W$ production and single diffractive production of jets with $p_{\mathrm{T}}$ thresholds of 20 and $50 \mathrm{GeV}$. Predictions according to PYTHIA 8.210 MBR [7] at $\sqrt{s}=14 \mathrm{TeV}$.

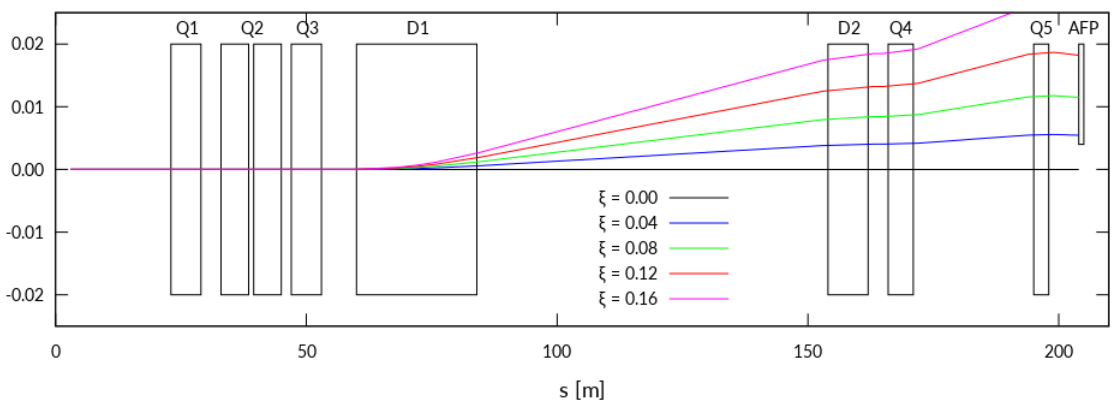

Fig. 2. Horizontal projections of trajectories of diffractively scattered protons through the LHC magnetic lattice.

It can be observed that not all of the scattered protons can be registered by the AFP detectors. The protons with too high energy loss are lost due to the LHC aperture, while those with too low energy loss are too close to the beam centre to be registered. In order to identify the kinematic region in which the detectors can perform the measurements, one often uses the plot of the acceptance as a function of $\xi$ and $p_{\mathrm{T}}$. An example of such a plot is presented in Fig. 3 (a). Two white areas, which mark the kinematic space where the measurements are not possible, can be seen. The lower region corresponds to protons too close to the beam, while the upper region reflects the influence of the LHC apertures. 


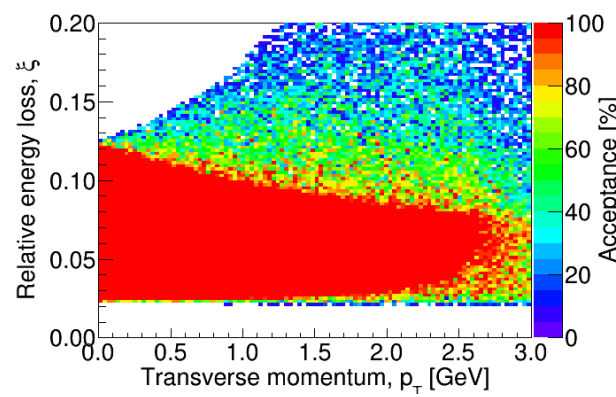

(a)

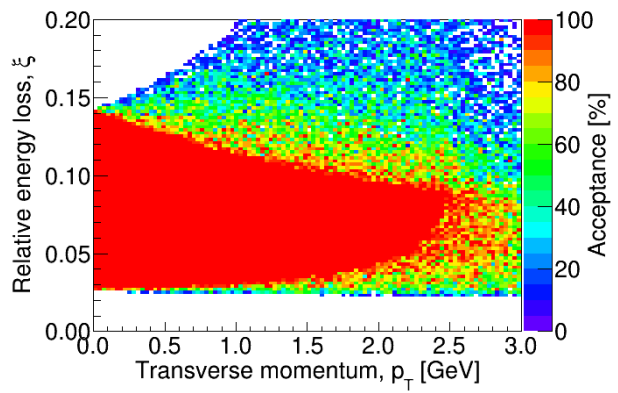

(b)

Fig. 3. The AFP acceptance as a function of the relative energy loss and the transverse momentum for the $\beta^{*}=0.55 \mathrm{~m}$ LHC optics for horizontal detectors at $205 \mathrm{~m}$ placed $3 \mathrm{~mm}$ from the centre of the beam. (a) Default optics, (b) Modified optics.

The acceptance plots presented in this paper were obtained in the following manner. At first, a set of 100000 protons was generated, with $p_{\mathrm{T}}, \varphi$ and $\xi$ distributed uniformly within ranges: $[0,3) \mathrm{GeV},[0,2 \pi)$ and $[0,0.2)$, respectively. Then, the generated protons were transported through the LHC lattice to the detector position using MAD-X. The acceptance in each $\left(p_{\mathrm{T}}, \xi\right)$ bin is calculated as the ratio of the number of protons hitting the detector to the number of generated protons. During the calculations, it was assumed that the detector is placed $3 \mathrm{~mm}$ from the centre of the beam.

One should note that the generated distributions of proton energy and transverse momentum are not physical. However, the acceptance is determined in bins of $\xi$ and $p_{\mathrm{T}}$. It is, therefore, averaged only over the $\varphi$ distribution, which is physically correct, because at the LHC, the beams are not polarised. The reason for the choice of unphysical distributions is that it allows the determination of the acceptance with small statistical uncertainties also in the phase-space regions where the diffractive protons are rare.

\section{Optics modifications}

One of the main factors affecting the acceptance of the AFP detectors are the properties of the LHC optics. This raises a question to what extent one could adjust the acceptance of the AFP detectors by modifying the optics. To study this problem, one needs to identify which elements of the LHC lattice have the largest influence on the trajectories of the diffractively scattered protons. As can be seen in Fig. 2, the acceptance is mainly determined by the D1 and D2 dipole magnets. Unfortunately, no changes of the dipole field strengths are possible (at a fixed value of $\sqrt{s}$ ), since the dipole magnets define the beam orbit. On the other hand, both the Q4 and Q5 
quadruple magnets have a non-negligible effect. Therefore, the influence of these two quadrupole magnets on the acceptance of the AFP detectors was checked.

In MAD-X, for each quadrupole magnet, one specifies the value of the quadrupole moment (the $k$-value). For the fourth and fifth quadruple magnet, the default values are: $k_{\mathrm{Q} 4}=-4.1 \mathrm{mT} / \mathrm{m}$ and $k_{\mathrm{Q} 5}=3.0 \mathrm{mT} / \mathrm{m}$, respectively. The allowed ranges of the modification of the Q4 and Q5 quadrupole moments are $(-0.15,-6.9) \mathrm{mT} / \mathrm{m}$ and $(0.16,6.9) \mathrm{mT} / \mathrm{m}$, respectively. Although in the present set-up, these magnets are designed to work with a given polarity of the voltage and a minimal current, in this study, the polarity was allowed to be reversed and the $k$-value to reach zero. This results in ranges of $(-6.9,6.9) \mathrm{mT} / \mathrm{m}$ for both considered quadrupoles.

To illustrate how the change of the Q4 and Q5 strengths can affect the acceptance, Fig. 3 presents a comparison of acceptances calculated with the design and a modified optics with the default $k_{\mathrm{Q} 4}$ value and $k_{\mathrm{Q} 5}=$ $6.9 \mathrm{mT} / \mathrm{m}$. One can see that the high-acceptance region extends towards the higher $\xi$ values and its $p_{\mathrm{T}}$ range is slightly shrunk.

High acceptance values, above $90 \%$, are obtained in a very wide range of the scattered proton transverse momentum, up to $p_{\mathrm{T}}=2.5 \mathrm{GeV}$. The real limitations for the measurements come from the limited $\xi$ acceptance. In order to see how this is affected by the machine optics changes, the acceptance as a function of $\xi$ was studied for $p_{\mathrm{T}}<0.3 \mathrm{GeV}$.

The upper and lower limits of the region with acceptance above $90 \%$ were determined for each studied optics. Figures 4 (a) and (b) present these limits as a function of the Q4 and Q5 strengths, respectively. It can be

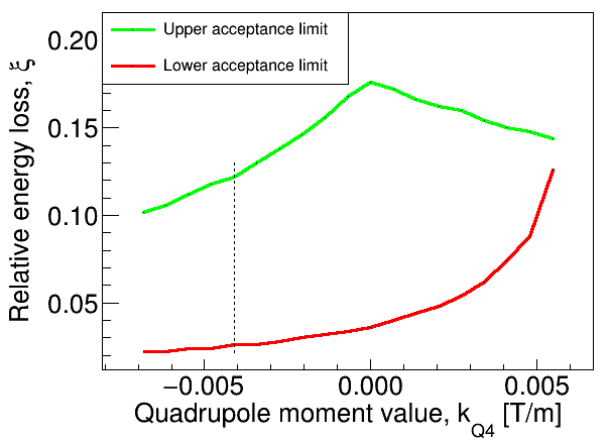

(a)

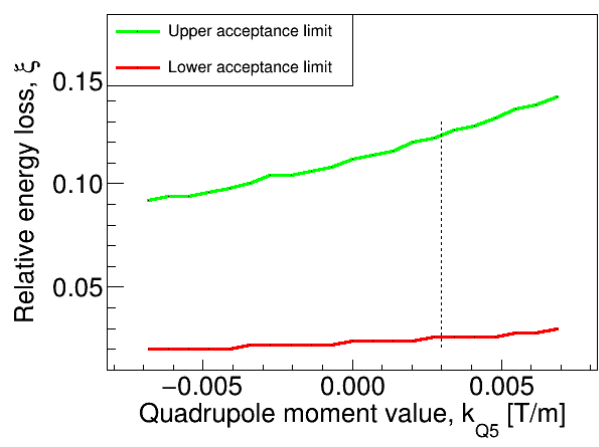

(b)

Fig. 4. Upper and lower acceptance limits as a function of (a) $k_{\mathrm{Q} 4}$, (b) $k_{\mathrm{Q} 5}$ for protons with $p_{\mathrm{T}}<0.3 \mathrm{GeV}$. The dashed lines mark the design optics. (a) Fourth quadrupole magnet, (b) Fifth quadrupole magnet. 
noticed that the Q4 magnet has a larger influence on the acceptance than the Q5 one. It is due to the fact that Q4 is placed further away from the AFP detectors than Q5. In addition, changing the $k$-values leads to a change of both limits in the same direction, i.e. when the lower limit increases, the upper limit also increases. The gain on one side is counterbalanced by the loss on the other side. However, this natural behaviour is not observed for reversed Q4 polarity, where different apertures come into play and decrease the high- $\xi$ acceptance.

\section{Summary}

The presented study shows that it is possible to change the AFP detectors acceptance by modifying the LHC optics, in particular by changing the value of the quadrupole moment of the Q4 and Q5 magnets. Unfortunately, the improvements are not large, and the gain at low $\xi$ values is associated with a loss at high $\xi$. Nevertheless, some flexibility is observed and it may become useful in the future. Finally, it must be pointed out that in the presented study it was not checked whether the assumed optics configurations can be matched to the rest of the LHC ring. On the other hand, since the considered changes do not require a modification of the final-focussing triplet settings, they should be feasible without a negative effect on the LHC luminosity.

We gratefully acknowledge Janusz Chwastowski for many stimulating discussions and for his comments to the manuscript. This work was supported in part by the Polish National Science Centre grant No. 2011/01/N/ ST2/03409.

\section{REFERENCES}

[1] L. Evans, P. Bryant, JINST 3, S08001 (2008).

[2] TOTEM Collaboration, JINST 3, S08007 (2008).

[3] CMS-TOTEM Collaboration, CMS-TOTEM Precision Proton Spectrometer, CERN-LHCC-2014-021, TOTEM-TDR-003, CMS-TDR-13.

[4] ATLAS Collaboration, ATLAS Forward Detectors for Measurement of Elastic Scattering and Luminosity, CERN-LHCC-2008-004. ATLAS-TDR-018,

[5] ATLAS Collaboration, Technical Design Report for the ATLAS Forward Proton Detector, CERN-LHCC-2015-009, ATLAS-TDR-024.

[6] MAD - Methodical Accelerator Design, https://mad.web.cern.ch/mad/

[7] T. Sjöstrand et al., Comput. Phys. Commun. 191, 159 (2015). 